\title{
Application of 2D-NMR with Room Temperature NMR Probes for the Assessment of the Higher Order Structure of Filgrastim
}

Robert G. Brinson ${ }^{a}$, Houman Ghasriani ${ }^{b, c}$, Derek J. Hodgson ${ }^{c}$, Kristie M. Adams ${ }^{d, \ddagger}$, Ian McEwen ${ }^{e}$, Darón I. Freedberg ${ }^{\mathrm{f}}$, Kang Chen ${ }^{\mathrm{b}}$, David A. Keire ${ }^{\mathrm{b}^{*}}$, Yves Aubin ${ }^{\mathrm{c}^{*}}$, and John P. Marino ${ }^{\mathrm{a}^{*}}$ 


\section{Abstract}

2 The higher order structure (HOS) of biotherapeutics is a critical quality attribute that can be

3 evaluated by nuclear magnetic resonance (NMR) spectroscopy at atomic resolution. NMR

4 spectral mapping of HOS can be used to establish HOS consistency of a biologic across

5 manufacturing changes or to compare a biosimilar to an innovator reference product. A previous

6 inter-laboratory study performed using filgrastim drug products demonstrated that two-

7 dimensional (2D)-NMR ${ }^{1} \mathrm{H}^{\mathrm{N}}-{ }^{15} \mathrm{~N}^{\mathrm{H}}$ heteronuclear correlation spectroscopy is a highly robust and

8 precise method for mapping the HOS of biologic drugs at natural abundance using high

9 sensitivity NMR 'cold probes.' Here, the applicability of the 2D-NMR method to fingerprint the

10 HOS of filgrastim products is demonstrated using lower sensitivity, room temperature NMR

11 probes. Combined chemical shift deviation and principal component analysis are used to

12 illustrate the performance and inter-laboratory precision of the 2D-NMR method when

13 implemented on room temperature probes.

16 Keywords: 2D-NMR, higher order structure, biologics, PCA, fingerprinting 


\section{Introduction}

The expiration of originator biologic drug patent protection and the advent of biosimilars has created a need for more advanced analytical methods to characterize the critical quality attributes (CQA) of these drug products that allow for robust demonstration of similarity. The higher order structure (HOS) of protein therapeutics, which is directly linked to both efficacy and safety of biologic drugs, is one of the CQAs that needs to be well characterized [1-4]. Originally proposed by Aubin and colleagues [5], precise and accurate assessment of the HOS of protein biotherapeutics, including monoclonal antibodies (mAbs) [6, 7], has been demonstrated using two dimensional nuclear magnetic resonance spectroscopy (2D-NMR). A single 2D-NMR heteronuclear correlation spectrum (e.g., $\left.{ }^{1} \mathrm{H}^{\mathrm{N}}-{ }^{15} \mathrm{~N}^{\mathrm{H}} \mathrm{HSQC}\right)$ can provide an assessment of

12 primary, secondary, tertiary and quaternary structure at atomic level resolution [8-11]. The cross-peaks in a 2D ${ }^{1} \mathrm{H}^{\mathrm{N}}-{ }^{15} \mathrm{~N}^{\mathrm{H}} \mathrm{HSQC}$ spectrum report on chemical shifts of individual protein backbone and side-chain amide ${ }^{15} \mathrm{~N}$ and ${ }^{1} \mathrm{H}$ nuclei. The composite pattern of a 2D-NMR spectral map affords a unique, well-dispersed pattern of cross-peaks, or so-called spectral fingerprint, since individual ${ }^{1} \mathrm{H}^{\mathrm{N}}-{ }^{15} \mathrm{~N}^{\mathrm{H}}$ resonances each report on their unique chemical and structural environments within the well-folded protein structure. Subsequently, any deviation from reference spectral positions of these cross peaks reflect a change in structure, assuming sample conditions have not deviated (e.g., temperature, salinity, $\mathrm{pH}$ ). Well matched 2D-NMR spectra therefore provide a high level of assurance that the predominant structure(s) of two protein biologic products (e.g., lot-to-lot, biosimilar to innovator) is highly similar.

Recently, Ghasriani et al. reported a study that evaluated the inter-laboratory precision

24 NMR cold probes [10]. One innovator filgrastim product (Neupogen $®$ ) and three Indian-sourced

25 products were evaluated in their fully formulated state. Filgrastim is prescribed to treat

26 neutropenia for cancer patients after chemotherapy for the stimulation of bone marrow to 
proliferate granulocytes such as neutrophils [12]. Filgrastim was chosen as a representative

2 protein therapeutic for this study as it had been previously well studied by NMR and other

3 biophysical methods [5, 13]. Filgrastim's primary structure consists of 175 amino acid residues,

4 including one extra methionine at the $\mathrm{N}$-terminus of the native 174-residue protein. Through the

5 inter-laboratory comparison, Ghasriani et al. found that 2D-NMR provides a highly robust and

6 reproducible measurement, and we established an inter-laboratory measurement precision of 8

7 parts per billion (ppb) for the method [10].

8 Here, a follow-up to the original inter-laboratory study is reported using a second set of

9 filgrastim drug products. While the precision of the NMR method is a function of the signal-to-

10 noise ratio [6], experimental protocols for the 2D-NMR method are shown that demonstrate how

11 it can be applied using moderate magnetic field strengths (e.g., $500 \mathrm{MHz}$ and $600 \mathrm{MHz}$ ) and

12 lower sensitivity, room temperature (RT) probes. The results show the robustness of the $2 \mathrm{D}$

13 method even when recorded on RT probes, and the use of rapid acquisition NMR methods

14 (e.g., non-uniform sampling, SOFAST) is more efficient application in biopharmaceutical HOS

15 assessment than standard acquisition [6]

\section{2. Materials and Methods}

\subsection{Formulated Drug Products}

Eight formulated filgrastim products were prepared for NMR measurements. Four of the

20 samples were from our previous study and will be referred to as lot 1 [10]. Four new samples,

21 herein referred to as lot 2, were purchased for this follow-up study: Amgen (Neupogen®, Lot:

22 1036977, Expiry: April 2015), Biocon (NUFIL Safe ${ }^{\mathrm{TM}}$, Batch number: N010069, Exp: Aug. 2014),

23 INTAS (Neukine®, B. No.: 1010072, PL. No.: G1010088, Expiry: June 2015) and Dr. Reddy's

24 Laboratories (Grafeel ${ }^{\mathrm{TM}}$, B. No.: GFAS01412, Expiry: October 2014). All samples were prepared

25 for NMR measurements at FDA laboratories as previously described [10]. Initial measurements

26 on the sample set were performed at NIST followed by USP and then FDA. 
2 2.2. NMR Instrumentation used for the acquisitions of $2 D-N M R$ data on the lot 2 samples Data were acquired at three different magnetic field strengths, $500 \mathrm{MHz}, 600 \mathrm{MHz}$, and

$4900 \mathrm{MHz}$ for ${ }^{1} \mathrm{H}$ resonance frequency using room temperature probes for detection at three

5 different laboratories. At $900 \mathrm{MHz}$, a cold probe was used. The laboratories were located at the

6 Food and Drug Administration (FDA), Center for Biological Evaluation and Research in

7 Bethesda, Maryland; United States Pharmacopeia (USP) in Rockville, Maryland; and the

8 National Institute of Standards and Technology (NIST), Institute for Bioscience and

9 Biotechnology Research in Rockville, Maryland. Table 1 lists all laboratories and samples (lot 1

10 and lot 2) used in the entire study. From the temperature deviant data in our first report, HC 600

11 spectra on lot 1 drug products were chosen as 'different' spectral maps for principal component 12 analysis (PCA).

\subsection{Experimental parameters for lot 2 samples}

All lot 2 drugs products were evaluated on the NIST 900 using a ${ }^{1} \mathrm{H}^{\mathrm{N}},{ }^{15} \mathrm{~N}^{\mathrm{H}}$ gradient selected heteronuclear single quantum coherence experiment (gHSQC) and all magnets

17 equipped with RT probes using a phase sensitive HSQC (pHSQC). All experiments were otherwise set-up as previously described with spectral widths of $14 \mathrm{ppm}\left({ }^{1} \mathrm{H}\right)$ by $32 \mathrm{ppm}\left({ }^{15} \mathrm{~N}\right)$, with the transmitters on water and $117 \mathrm{ppm}$, respectively [10]. Acquisition time was set to 140 ms (NIST 900), 100 ms (NIST RT600, USP RT600), or 85 ms (FDA RT500), and 64 complex

21 points were collected in the ${ }^{15} \mathrm{~N}$ dimension. The interscan delay was set to $1.0 \mathrm{~s}$ (1.5 s NIST 22 900). Total experimental time was $62 \mathrm{~h}$ (NIST 900) and approximately $165 \mathrm{~h}$ for all instruments 23 with RT probes. The FDA RT500 spectra were recorded as nine experiments that were co24 added within Topspin 3.2 (Bruker Biospin, Inc) before spectral processing. 
Spectral analysis. All data for drug products were processed or reprocessed at NIST using

$2 \quad$ NMRPipe V8.9 [14] using a similar scheme as reported previously [10]. Data were visualized in

3 NMRFAM-Sparky V1.2 [15]. All spectra were aligned to glycine 73 at $7.77 \mathrm{ppm}\left({ }^{1} \mathrm{H}\right)$ and 105.8

$4 \quad \mathrm{ppm}\left({ }^{15} \mathrm{~N}\right)$. Signal-to-noise $(\mathrm{S} / \mathrm{N})$ values were determined in NMRFAM-Sparky.

$5 \quad$ For PCA, lot 1 and lot 2 drug products were bulk converted to NMRPipe format and bulk

6 processed using scripts embedded within NMRPipe V8.9 to assure uniform conversion and

7 processing. The processing parameters from our first report were used [10]. Imperfect phasing

8 of any spectra was corrected manually. For PCA on peak position, all resolved ${ }^{1} \mathrm{H}^{\mathrm{N}},{ }^{15} \mathrm{~N}^{\mathrm{H}}$ cross

9 peaks were used (roughly $60 \%$ ), and the chemical shifts were weighted according to:

10 and Machine Learning Toolbox ${ }^{\mathrm{TM}}$ in MATLAB R2015b (Mathworks, Inc). was performed. ppm to $132 \mathrm{ppm}$ for ${ }^{15} \mathrm{~N}$ ), a uniform matrix size for all data is required for any multivariate

where $\delta_{H}$ and $\delta_{N}$ are the ${ }^{1} \mathrm{H}$ and ${ }^{15} \mathrm{~N}$ chemical shifts for each amide, and $\alpha$ is 0.1 . The weighted chemical shifts for each resonance from the NIST 900 Amgen lot 2 spectrum were subtracted from each dataset (e.g., the position is centered by subtracting the column means) before PCA

For PCA computed on the entire amide region (5.75 ppm to $10.5 \mathrm{ppm}$ for ${ }^{1} \mathrm{H}$ and 103 analysis, so all data were subjected to a bilinear interpolation scheme, also embedded within NMRPipe V8.9, to create a uniform matrix size for all spectra, and intensities were normalized to 100 for the most intense peak within each spectrum. The amide region of each spectrum was then converted to $1 \mathrm{D}$ intensity vectors. PCA was directly applied to the raw data in this case to avoid overemphasizing the noise which would occur by taking a column-centered means. In both applications of PCA, the single value decomposition algorithm was used from the Statistics 


\section{3. Results and Discussion}

\section{3.1. Visual Assessment of 2D Spectral Maps}

The second lots of the innovator and three Indian-sourced filgrastim products in their

4 formulated states were evaluated using the NIST 900 equipped with a NMR cold probe and

5 three magnets equipped with room temperature (RT) probes (Figures 1, S1 - S3). The time

6 required to acquire data of sufficient quality (similar resolution and a minumum S/N ratio of 10:1)

7 took about 2.7 times longer on the RT probes when compared to the $900 \mathrm{MHz}$ data (62 $\mathrm{h}$ on the

8 NIST 900 versus $165 \mathrm{~h}$ on the RT probes). To acquire a dataset of similar S/N ratio on a RT

9 probe would require 16 to 36 times the acquisition time as compared to a comparable cold

10 probe assuming a factor of 4 to 6 sensitivity gain from the cold probe. Despite the lower $\mathrm{S} / \mathrm{N}$

11 ratio, an initial visual inspection of the RT spectra shows almost perfect spectral overlap with

12 the $900 \mathrm{MHz}$ data, indicating that all the 2D NMR datasets report the same highly similar HOS

13 for the samples. Note that some spectral variation was observed due to field dependent spectral

14 folding of few side chain resonances.

3.2. Spectral Comparability using Combined Chemical Shift Deviation and Principal Component

17 Analysis

In a manner similar to the original report, ${ }^{1} \mathrm{H}$ and ${ }^{15} \mathrm{~N}$ chemical shifts of the drug products were evaluated using combined chemical shift deviation (CCSD) referenced against the NIST 900 Amgen lot $2 \mathrm{gHSQC}$ (Figures 2, S4) or against the NIST 600 Amgen lot 2 gHSQC (Figure

21 S5). In both analyses, the overall CCSD precision of the measurements was found to be highly 22 similar whether referenced again cold probe data collected at $900 \mathrm{MHz}$ or $600 \mathrm{MHz}$. In general, 23 most spectral maps of the lot 2 products collected on magnets equipped with cold probes fell 24 within previously established experimental precision of 8 parts per billion (ppb) (Figure S4D) 25 [10]. The RT spectral maps exhibited slightly greater chemical shift deviation, consistent with a 26 previous report that $\mathrm{S} / \mathrm{N}$ below 10:1 affords a reduction in statistical precision [6]. For 
comparison, in this study the NIST 900 Amgen spectral map had a mean $S / N$ of $36: 1$ and a median S/N of 25:1 whereas the NIST RT600 Amgen spectral map had values of 10:1 and 7:1, respectively. Due to the lower $\mathrm{S} / \mathrm{N}$, the RT spectral maps exhibited a precision of approximately $16 \mathrm{ppb}$ which was only slightly lower when compared to the spectral precision measured with cold probes. The RT spectra collected in the non-conventional manner of nine co-added experiments (see Material \& Methods section), however, afforded a few resonances with precision less than $16 \mathrm{ppb}$.

To assess the comparability of lot 2 samples versus the lot 1 samples, PCA was performed on the weighted ${ }^{1} \mathrm{H}$ and ${ }^{15} \mathrm{~N}$ chemical shifts of the temperature calibrated spectral maps (Figure 3A) and the total point-by-point spectral region akin to our previous report (Figures 3B and S6) [10]. The temperature-miscalibrated HC 600 spectral maps were used as an example of a slightly 'different' spectra to help evaluate the PCA clustering. Both PCA plots show similar clustering of the lot 2 spectral data with the lot 1 spectral data, and the HC 600 data separated as its own cluster. Overall, these results confirm that standard RT probes can offer a robust means to evaluate the HOS of biologics.

\subsection{Rapid NMR data acquisition methods.}

Rapid NMR data acquisition protocols can be applied to markedly reduce the acquisition time for 2D NMR spectra [9]. As a demonstration, spectra were collected using the SOFASTHMQC pulse scheme (sfHMQC) [16] on Amgen lot 1 on the NIST 900 and Amgen Lot 2 on the NIST RT600 (Figure S7). Slight visual differences were observed due to slight differences in the performance of the gHSQC and the sfHMQC pulse sequences. Nonetheless, rapid pulsing cut the overall acquisition times on the NIST 900 and the NIST RT600 to $13 \mathrm{~h}$ and $72 \mathrm{~h}$, respectively. Further reductions in overall experimental time could be achieved by implementing non-uniform sampling (NUS), which is also becoming more routinely applied for NMR acquisitions. For this comparative study, acquisition methods were nonetheless kept to standard 
1 pulse sequences with uniform sampling to establish an unbiased baseline for judging the

2 performance of RT probes for use in biopharmaceutical comparability measurements. Based on

3 the performance differences observed for different pulse sequences, the choice of HSQC

4 experiment should be consistently applied for the 2D-NMR method, similar to the requirement

5 for the same temperature, $\mathrm{pH}$, and salinity.

\section{Conclusions}

For a technique that generates a spectral map that simultaneously yields information

9 about primary, secondary, tertiary and higher order structure, 2D-NMR provides a precise tool to

10 assess drug substance quality for sponsors of biopharmaceuticals. While collecting 2D-NMR

11 heteronuclear correlation maps at natural abundance on NMR systems equipped with cold

12 probes is ideal for sensitivity, this study demonstrates that 2D-NMR spectral maps can be

13 generated with similar quality using NMR systems with RT probes and that these data can be

14 used for comparability assessment. With the use of SOFAST pulse sequences and NUS [7, 17]

15 that can generate greater sensitivity per unit time, $2 \mathrm{D}{ }^{1} \mathrm{H}^{\mathrm{N}},{ }^{15} \mathrm{~N}^{\mathrm{H}}$ spectral maps should be able to 16 be collected on RT probes in 2 to 3 days.

\section{Disclaimers}

This article reflects the views of the author and should not be construed to represent U.S. FDA's views or policies.

Certain commercial equipment, instruments, and materials are identified in this paper in

22 order to specify the experimental procedure. Such identification does not imply recommendation

23 or endorsement by the National Institute of Standards and Technology, nor does it imply that the 24 material or equipment identified is necessarily the best available for the purpose.

\section{Acknowledgements}


We acknowledge the support by NIST Biomanufacturing Initiative and NIST and W.M.

2 Keck for support of Biomolecular NMR instrumentation. Additional support for this work was, in

3 part, from the US FDA Critical Path funding.

4 Appendix A. Supplementary Data

Supplementary data associated with this article can be found in the online version.
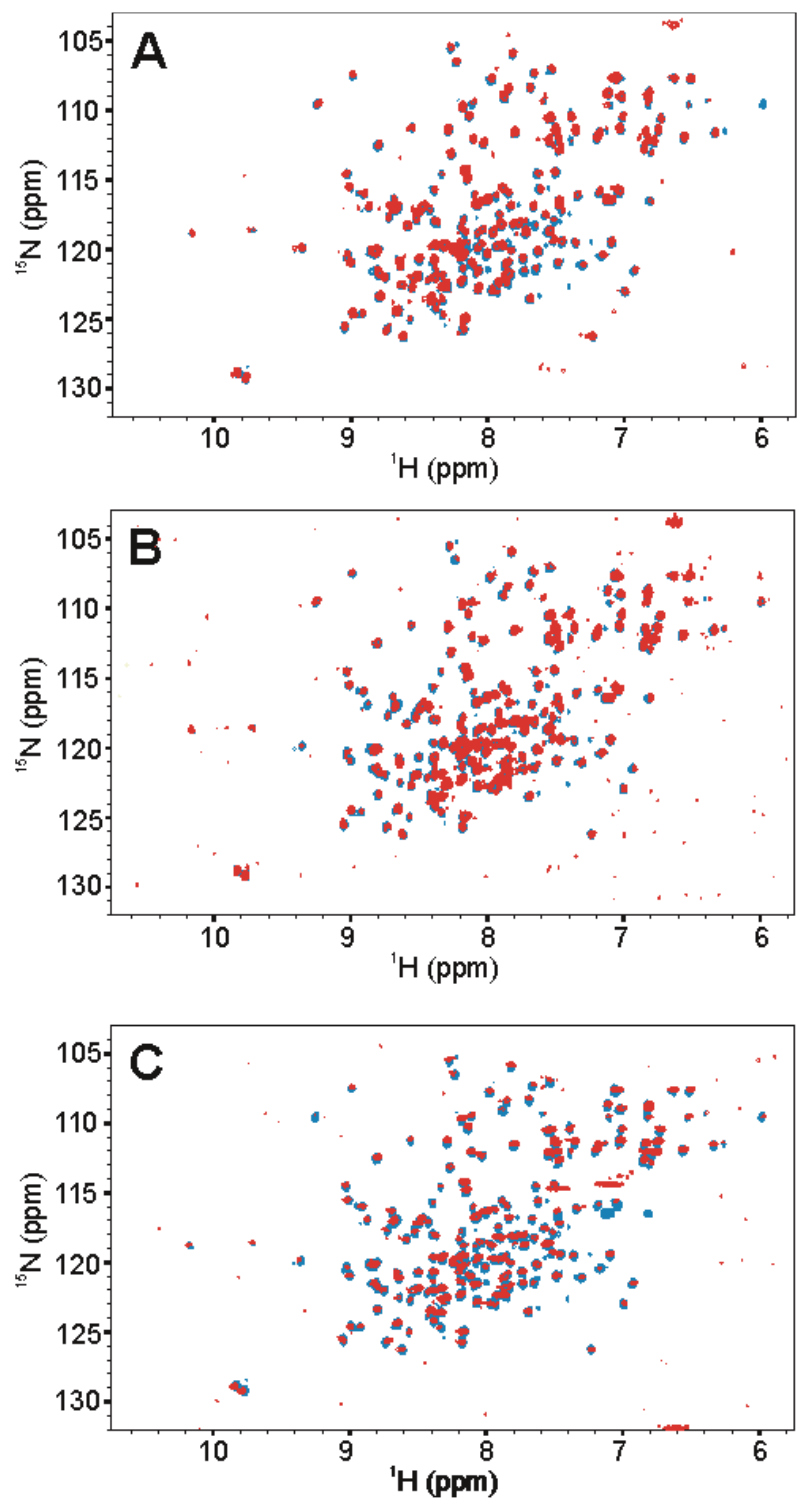

6 
1 Figure 1. Amide fingerprint region of representative $\mathrm{RT}{ }^{1} \mathrm{H}^{\mathrm{N}}, \mathrm{N}^{\mathrm{H}}$ spectral maps of Amgen lot 2, 2 in red, overlaid on NIST900 Amgen lot 2 product in blue. (A) NIST RT600; (B) USP RT600; (C) 3 FDA RT500. All experiments were recorded using a gHSQC pulse sequence at $25^{\circ} \mathrm{C}$. Contour $4 \quad$ levels were chosen at the noise threshold. 


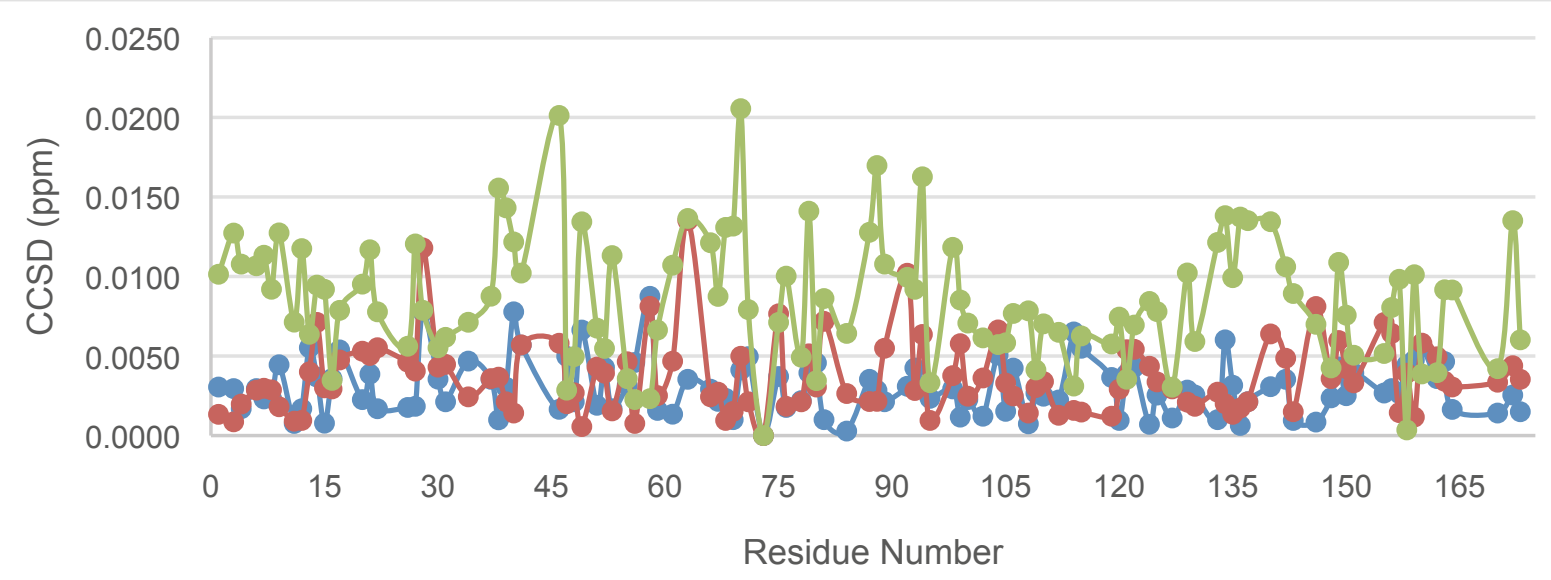

3

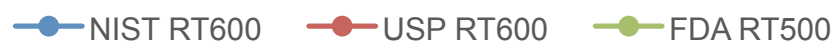

4

5 Figure 2. Representative CCSD plot of Amgen lot 2 RT spectra. The resonance positions from

6 the NIST 900 Amgen lot $2 \mathrm{gHSQC}$ were used as a reference. The observed higher CCSD for

7 the FDA RT500 is likely due to a slightly different acquisition protocol. See Materials \& Methods

8 for details. Other CCSD sample data are given in the Supporting Information (Figure S4).

9

10 


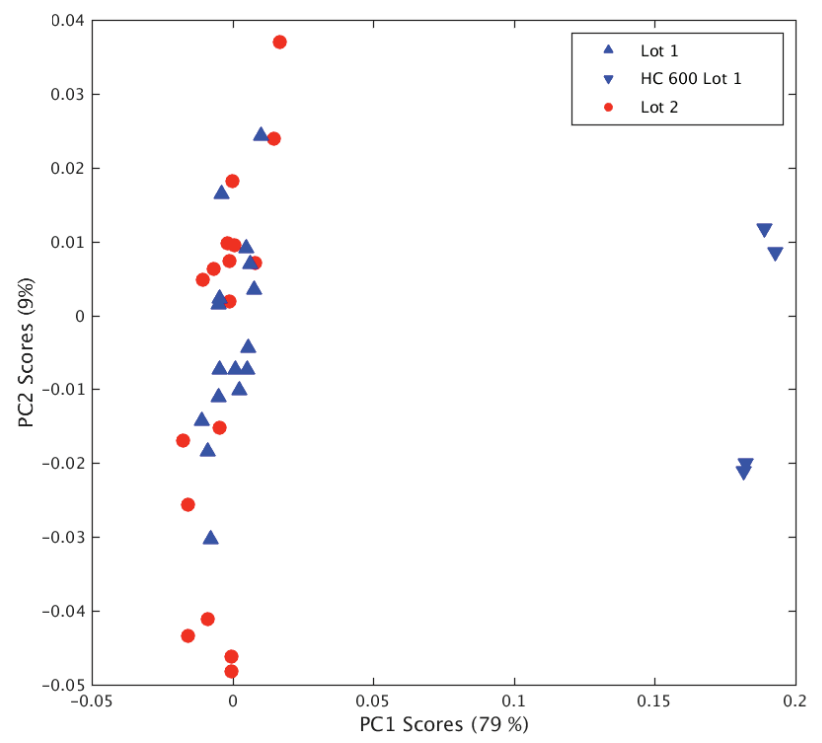

3B

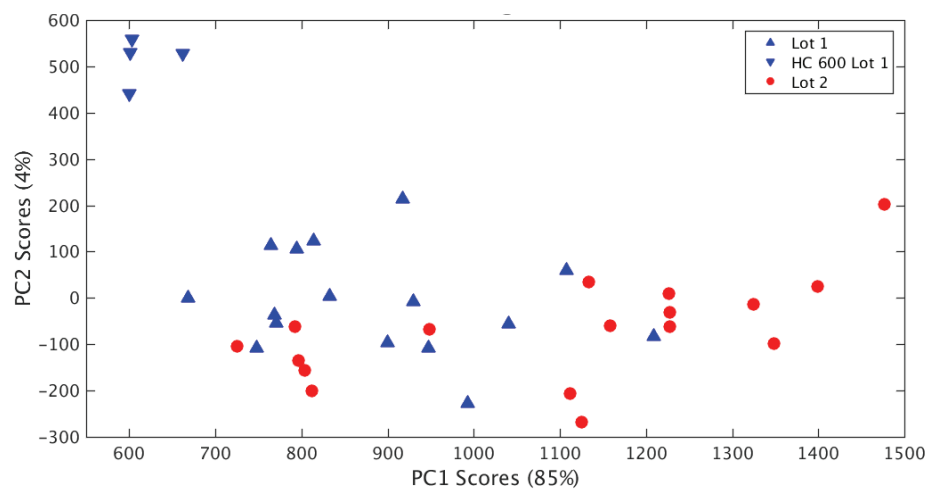

16 Figure 3. PCA Plots by 2 Different Methods. (A) PCA plot on the combined weighted ${ }^{1} \mathrm{H}$ and ${ }^{15} \mathrm{~N}$

17 chemical shifts of resolved peaks. (B) PCA plot of the total point-by-point spectral matrix of the

18 amide fingerprint region. In both PCA plots, the HC 600 temperature deviant spectra from our

19 first report were used as 'different' spectra for comparative purposes [10]. For clarity, the

20 projections in PC space are labeled by sample. For panel $\mathrm{B}$, a HSQC is a matrix of frequency-

21 based based with intensity values. These spectral matrices were converted to 1D intensity

22 vectors, and all vectors were subjected to PCA. See Material and Methods section for more

23 details. Additional annotations of panel B are available in Figure S6. 
2 Table 1. Equipment type and experiments performed on each sample

\begin{tabular}{|l|l|l|l|l|l|l|}
\hline $\begin{array}{l}\text { Short } \\
\text { hand }\end{array}$ & Lab & $\begin{array}{l}{ }^{1} \mathbf{H} \\
\text { Frequency } \\
\text { (MHz) }\end{array}$ & Console & Probe & Experiment & $\begin{array}{l}\text { Sample(s) } \\
\text { analyzed }\end{array}$ \\
\hline $\begin{array}{l}\text { NIST } \\
\mathbf{9 0 0}\end{array}$ & NIST & 900 & $\begin{array}{l}\text { Bruker } \\
\text { Avance III }\end{array}$ & $\begin{array}{l}\text { TCl } \\
\text { cryoprobe }\end{array}$ & gHSQC & lot 1, lot 2 \\
\hline $\begin{array}{l}\text { NIST } \\
\mathbf{6 0 0}\end{array}$ & NIST & 600 & $\begin{array}{l}\text { Bruker } \\
\text { Avance III }\end{array}$ & $\begin{array}{l}\text { TXI or TCl } \\
\text { cryoprobe }\end{array}$ & gHSQC & lot $1^{1}$, lot $2^{2}$ \\
\hline $\begin{array}{l}\text { NIST } \\
\text { RT600 }\end{array}$ & NIST & 600 & $\begin{array}{l}\text { Bruker } \\
\text { Avance I }\end{array}$ & TXI & pHSQC & lot 2 \\
\hline HC 700 & HC & 700 & $\begin{array}{l}\text { Bruker } \\
\text { Avance I }\end{array}$ & $\begin{array}{l}\text { TCl } \\
\text { cryoprobe }\end{array}$ & gHSQC & lot 1 \\
\hline HC 600 & HC & 600 & $\begin{array}{l}\text { Bruker } \\
\text { Avance III }\end{array}$ & $\begin{array}{l}\text { TCl } \\
\text { cryoprobe }\end{array}$ & gHSQC & lot 1 \\
\hline $\begin{array}{l}\text { MPA } \\
\mathbf{6 0 0}\end{array}$ & MPA & 600 & $\begin{array}{l}\text { Bruker } \\
\text { Avance I }\end{array}$ & $\begin{array}{l}\text { TCl } \\
\text { cryoprobe }\end{array}$ & pHSQC & lot 1 \\
\hline $\begin{array}{l}\text { USP } \\
\text { RT600 }\end{array}$ & USP & 600 & $\begin{array}{l}\text { Bruker } \\
\text { Avance III }\end{array}$ & TXI & pHSQC & lot 2 \\
\hline $\begin{array}{l}\text { FDA } \\
\text { 500 }\end{array}$ & FDA $^{3}$ & 500 & $\begin{array}{l}\text { Agilent } \\
\text { Direct Drive }\end{array}$ & Cryobay VS & gHSQC & lot 1 \\
\hline $\begin{array}{l}\text { FDA } \\
\text { RT500 }\end{array}$ & FDA $^{4}$ & 500 & $\begin{array}{l}\text { Bruker } \\
\text { Avance III }\end{array}$ & QXI & pHSQC & lot $2^{5}$ \\
\hline
\end{tabular}

3

$4 \quad{ }^{1}$ Data were collected with TXI cryoprobe

$5 \quad{ }^{2}$ Data were collected on Amgen and Dr. Reddy lot 2 samples with a TCl cryoprobe.

$6{ }^{3}$ Laboratory located in St. Louis, MO, USA.

$7 \quad{ }^{4}$ Laboratory located in Bethesda, MD, USA.

$8 \quad{ }^{5}$ No experiment was collected for Dr. Reddy lot 2 sample. 
References

[1] O.V. Borisov, J.E. Schiel, D. Davis, Trends and Drivers for the Development of NextGeneration Biotherapeutic Characterization Tools, in: J.E. Schiel, D.L. Davis, O.V. Borisov (Eds.), State-of-the-Art and Emerging Technologies for Therapeutic Monoclonal Antibody Characterization, Vol 3: Defining the Next Generation of Analytical and Biophysical Techniques, American Chemical Society, Washington, 2015, pp. 1-16.

[2] J.P. Gabrielson, W.F. Weiss, Technical Decision-Making with Higher Order Structure Data: Starting a New Dialogue, J. Pharm. Sci. 104 (2015) 1240-1245.

[3] W.F. Weiss, J.P. Gabrielson, W. Al-Azzam, G. Chen, D.L. Davis, T.K. Das, D.B. Hayes, D. Houde, S.K. Singh, Technical Decision Making With Higher Order Structure Data: Perspectives on Higher Order Structure Characterization From the Biopharmaceutical Industry, J. Pharm. Sci. 105 (2016) 3465-3470.

[4] A.C. Fisher, S.L. Lee, D.P. Harris, L. Buhse, S. Kozlowski, L. Yu, M. Kopcha, J. Woodcock, Advancing pharmaceutical quality: An overview of science and research in the U.S. FDA's Office of Pharmaceutical Quality, Int. J. Pharm. 515 (2016) 390-402.

[5] Y. Aubin, G. Gingras, S. Sauve, Assessment of the three-dimensional structure of recombinant protein therapeutics by NMR fingerprinting: demonstration on recombinant human granulocyte macrophage-colony stimulation factor, Anal. Chem. 80 (2008) 2623-2627.

[6] L.W. Arbogast, R.G. Brinson, T. Formolo, J.T. Hoopes, J.P. Marino, 2D (1)H(N), (15)N Correlated NMR Methods at Natural Abundance for Obtaining Structural Maps and Statistical Comparability of Monoclonal Antibodies, Pharm. Res. 33 (2016) 462-475.

[7] L.W. Arbogast, R.G. Brinson, J.P. Marino, Mapping Monoclonal Antibody Structure by 2D ${ }^{13} \mathrm{C}$ NMR at Natural Abundance, Anal. Chem. 87 (2015) 3556-3561.

[8] Y. Aubin, D.I. Freedberg, C. Jones, Using NMR Spectroscopy to Obtain the Higher Order Structure of Biopharmaceutical Products: Simple Methods Can Characterize Polysaccharide Vaccines and Recombinant Cytokines at High Resolution, Biopharm Int. (2010) S28+.

[9] L.W. Arbogast, R.G. Brinson, J.P. Marino, Application of Natural Isotopic Abundance (1)H(13)C- and (1) $\mathrm{H}-(15) \mathrm{N}$-Correlated Two-Dimensional NMR for Evaluation of the Structure of Protein Therapeutics, Methods Enzymol. 566 (2016) 3-34.

[10] H. Ghasriani, D.J. Hodgson, R.G. Brinson, I. McEwen, L.F. Buhse, S. Kozlowski, J.P. Marino, Y. Aubin, D.A. Keire, Precision and robustness of 2D-NMR for structure assessment of filgrastim biosimilars, Nat. Biotechnol. 34 (2016) 139-141.

[11] B. Japelj, G. Ilc, J. Marusic, J. Sencar, D. Kuzman, J. Plavec, Biosimilar structural comparability assessment by NMR: from small proteins to monoclonal antibodies, Sci. Rep. 6 (2016) 32201.

[12] L.M. Souza, T.C. Boone, J. Gabrilove, P.H. Lai, K.M. Zsebo, D.C. Murdock, V.R. Chazin, J. Bruszewski, H. Lu, K.K. Chen, J. Barendt, E. Platzer, M.A.S. Moore, R. Mertelsmann, K. Welte, Recombinant human granulocyte colony-stimulating factor - effects on normal and leukemic myeloid cells, Science 232 (1986) 61-65.

[13] T. Zink, A. Ross, K. Luers, C. Cieslar, R. Rudolph, T.A. Holak, Structure and dynamics of the human granulocyte-colony-stimulating factor determined by nmr-spectroscopy - loop mobility in a 4-helix-bundle protein, Biochemistry 33 (1994) 8453-8463. 
1 [14] F. Delaglio, S. Grzesiek, G.W. Vuister, G. Zhu, J. Pfeifer, A. Bax, NMRPipe: a 2 multidimensional spectral processing system based on UNIX pipes, J. Biomol. NMR 6 (1995) $3 \quad 277-293$.

4 [15] W. Lee, M. Tonelli, J.L. Markley, NMRFAM-SPARKY: enhanced software for biomolecular $5 \quad$ NMR spectroscopy, Bioinformatics 31 (2015) 1325-1327.

6 [16] P. Schanda, B. Brutscher, Very fast two-dimensional NMR spectroscopy for real-time 7 investigation of dynamic events in proteins on the time scale of seconds, J. Am. Chem. Soc. 8127 (2005) 8014-8015.

9 [17] M.R. Palmer, B.R. Wenrich, P. Stahlfeld, D. Rovnyak, Performance Tuning Non-uniform 10 Sampling for Sensitivity Enhancement of Signal-limited Biological NMR, J. Biomol. NMR 58 11 (2014) 303-314. 